\title{
ENDOGENOUS HORMONAL STATUS OF EMBRYOGENIC CALLUS IN COCOS (Cocos nucifera L.) EXPLANTS
}

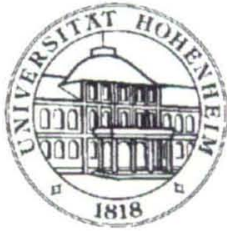

INTRODUCTION

The application of optimum concentrations of hormones according to specific culture stages plays an important role, especially in plants which are concerned to be recalcitrant for propagation in vitro, like cacos nucifera L. The feasibility of the clonal propagation of coconut via somatic embryogenesis using immature inflorescences as source of explants has been demonstrated by Branton and Blake (1984). But yet there is no advanced technique for the mass production of clonal palms available. One reason among others might be the inconsistent in vitro responses of coconut cultures observed in various clonal propagation experiments (Ebert, 1993).

The present study was done to elucidate the endogenous hormonal changes in coconut explants, focusing on the 4-months period of embryogenic callus formation in rachilla explant cultures.

\section{MATERIALS AND METHODS}

Rachillae derived from immature inflorescences of the Cocos nucifera $L$ c cultivar Laguna Tall were used as explant source. The rachillae were divided into tip, middle and basal section and then sliced transversely $(0.5-1 \mathrm{~mm})$. The explants were cultured onto semi-solid medum (Branton and Blake, 1984) supplemented with $22.1 \mathrm{mg} /$ (week 0-4) and 44.2mgl (week 4-16) 2,4-dichiorophenoxyacetic acid (2,4-D) in combination with $0.2 \mathrm{mgl}$ (week 0-16) isopentenyladenine (ZiP). After a visual assessment for browning and callus formation the explants were transferred onto fresh medium every 4 weeks. Formation of a globular, white callus became visble 8 to 10 weeks after initiation. Using radoimmunoassay (RIA) the endogenous concentrations of the cytokinins zeatin/zeatinniboside (Z/ZR) and isopentenyladenosine/-adenine (IP/IPA) as well as of free indole-3-acetic acid (IAA) and abscisic acid (ABA) were quantified.

Data for free IAA and ABA are not shown here

\section{RESULTS}

Measurements at 4 week intervals (Fig. 1), e.g. at every subculture, indicated that the main hormonal changes occur similarly within the first 4 weeks of culturing in vitro in all sections of the rachillae. Compared to the initial hormonal status of the explants the endogenous concentrations of ZZZ (Fig. 1a) and ABA decreased whereas IP/IPA (Fig. 1b) and free IAA level mostly increased until week 4. Prolonged growth of the rachilla explants was not possible on hormone-free medurn. In some experiments a slight increase of the ZZR concentration was noticeable between week 12 and 16 when callus formation alreacy had become visible (data not shown). Determination of the endogenous Z/ZR concentration in callus, explants with callus formation and explants lacking callus formation in week 16 (Fig. 2a) indicated higher ZZR levels in callus bearing explants. The same pattern could be observed by quantification of the endogenous IPAPA (Fig. 2b). Lowest percentage of callus formation could regularty be observed in the basal section of the rachillae.

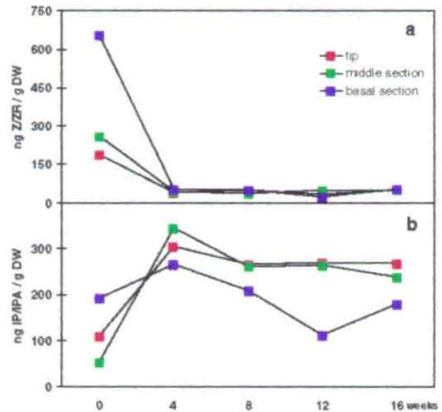

\begin{tabular}{|l|ccc|}
\hline \multicolumn{4}{|c|}{ callus formation (8) } \\
\hline weeks & 8 & 12 & 16 \\
\hline tip & 4.3 & 9.6 & 10.0 \\
middle section & 0.9 & 70 & 5.5 \\
basal section & 0.0 & 0.0 & 0.0 \\
\hline
\end{tabular}

Fig. 1. Endogenous ZZZR and IPIPA concentrations (ng/g dry weight) and callus tormation ( $(\%)$ in rachilla expiants of the Cocos nucifera $L$ cultiva Laguna Tall during the 4-months callus induction period grown on medum supplemented with 2,4D and 2ip

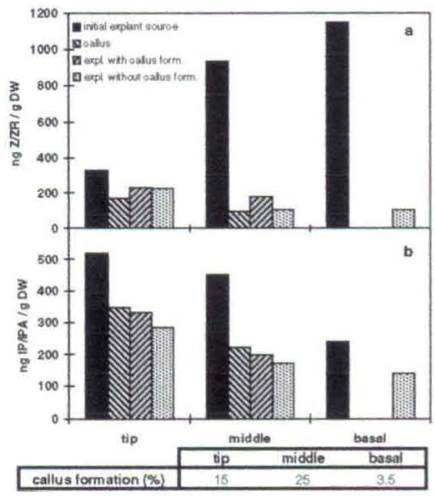

Fig 2. Endogenous ZIZA and IPIPA concentrations (ng/g dry weight) and callus formation $(\%)$ in ractilla explants of the Cocos nucilera $L$ culviver Lagung Tall in the beginning and atter 16 weeks (expients seperated into callus and explants with or without calus formation) grom on medium supdemented with 2 , D and or

\section{CONCLUSIONS}

In cocos explant cultures added exogenous auxins and cytokinins are associated with promotion of growth and callus induction. In addition, there is much evidence that the presence of exogenous plant growth regulators in the meda is necessary to maintain certain endogenous hormone levels in the explants.

The results of the quantification of endogenous cytokinin concentrations and data obtained from other experiments seem to indicate that the conversion from isopentenyladenosine to zeatin is blocked during the first weeks of culture. When callus formation starts this blockade in the zeatin biosynthesis pathway might have been already overcome.

The data for endogenous IPIPA and free IAA during culture of rachilla explants and in callus forming explants point at a possible interaction between these hormones. High IPIPA levels and decreased free IAA concentrations were detected in culture explants showing a high amount of callus. Therefore, there might be a response of the explant tissues in terms of callus formation to a balance or ratio of IP/PA and free IAA. 